\title{
Exclusive rare B-decays in the heavy quark limit
}

\author{
A. Ali and T. Mannel ${ }^{1}$ \\ Deutsches Elektronen Synchrotron DESY, W-2000 Hamburg, FRG
}

Received 30 March 1991; revised manuscript received 17 May 1991

\begin{abstract}
We calculate the rare $\mathrm{B}$-decays $\mathrm{B} \rightarrow \mathrm{K}^{*} \gamma$ and $\mathrm{B} \rightarrow\left(\mathrm{K}, \mathrm{K}^{*}\right), \bar{\ell}(\ell=\mathrm{e}, \mu, v)$ in the limit when the quark masses $m_{\mathrm{b}}, m_{\mathrm{c}}$ and $m_{\mathbf{s}}$ are considered heavy. In this limit, the semileptonic decays $B \rightarrow D \ell v_{\ell}, B \rightarrow D^{*} \ell v_{\ell}, D \rightarrow K \ell v_{\ell}, D \rightarrow K^{*} \ell v_{\ell}$ and the mentioned rare $B$-decays are related. Experimental data on the semileptonic decays then provide both input information for the rare B-decays as well as an estimate of the error of our approximation.
\end{abstract}

\section{Introduction}

Rare B-decays, in particular those involving $b \rightarrow s \gamma$ and $b \rightarrow s \ell \bar{\ell}(\ell=e, \mu, \tau, v)$ transitions at the quark level, provide an important testing ground for the standard model and hence they have received a lot of theoretical attention [1-6]. Compared to the corresponding K-decays, which are also an important source of information on the flavour changing neutral current transitions, the much disputed long distance effects are expected to be either suppressed in B-decays, due to the larger mass of the b-quark, or else can be removed by appropriate kinematic considerations. This aspect then reduces, in principle, one potential source of ambiguity in estimating the strength of the relevant effective hamiltonian.

To predict the exclusive decay rates there still remains the task of evaluating the hadronic matrix elements of the effective operators, and various model dependent approaches have been developed to estimate them [6-10]. In this context we note that significant progress has been made recently in developing an effective field theory governing the heavy hadron decays, by using a static limit for the heavy quarks in QCD [11-13]. In general, the static limit may be regarded as the first term of a systematic $1 /$ $m_{\mathrm{Q}}$ expansion, where $m_{\mathrm{Q}}$ is the quark mass. The heavy quark effective field theory has a lot of predictive power, though prima facie the applications are re-

\footnotetext{
1 Supported by a grant from Deutsche Forschungsgemeinschaft.
}

stricted to the decays in which both the decaying and the final state quarks (hadrons) are heavy. It may, however, be possible to systematically classify the leading $1 / m_{\mathrm{Q}}$ corrections [13], and for some specific processes the first order corrections have indeed been studied [14].

In this paper we work in the static limit and study the rare transitions $B \rightarrow K^{*} \gamma$ and $B \rightarrow\left(K, K^{*}\right) \ell \bar{\ell}(l=$ $e, \mu, v)$. Assuming that the s-quark is heavy, we may relate these rare decays to the semileptonic decays of the D- and B-mesons. Very definitely, the s-quark is not heavy and $1 / m_{\mathrm{s}}$ corrections need to be included in order to obtain reliable results. Despite this, it is tempting to use the static limit to predict the absolute decay rates for $D \rightarrow K^{(*)} \ell v_{\ell}, D \rightarrow K \ell v_{\ell}$ from $B \rightarrow D^{(*)} \ell v_{\ell}$. This exercise yields results which are in agreement with data within a factor of two (see below). It is, therefore, not an unreasonable hope that the static limit may provide results of a comparable accuracy also for the rare B-decays. In any case, the results presented here correspond to a well defined limit and they may encourage attempts to calculate the leading correction in $1 / m_{\mathrm{s}}$ in the effective field theory approach.

In section 2 we define the effective hamiltonian including the QCD corrections [3]. The various decay rates of interest are calculated in section 3. First, we briefly review the calculation of the hadronic matrix elements in the heavy quark limit, then apply the formalism to the semileptonic decays $\mathrm{D} \rightarrow \mathrm{K} \ell v_{\ell}$ and $\mathrm{D} \rightarrow \mathrm{K}^{*} \ell v_{\ell}$ and compare the results with data. From 
these we predict the rates for the exclusive rare decays of the B-meson, $B \rightarrow K^{*} \gamma$, and $B \rightarrow\left(K, K^{*}\right) \ell \bar{\ell}$ $(\ell=e, \mu, v)$. We present the invariant missing mass distributions, resulting from the decays $B \rightarrow(K$, $\left.\mathrm{K}^{*}\right) \bar{v} \bar{v}$ and the inclusive rare decays $\mathrm{B} \rightarrow \mathrm{X}_{\mathrm{s}} v \bar{v}$, where $X_{\mathrm{s}}$ denotes any strange hadronic state with strangeness $S=-1$. These distributions may become accessible in the decays $Z^{0} \rightarrow b \bar{b}$, in high luminosity LEP experiments. The invariant mass distributions involving the charged dileptons will be presented in a forthcoming publication, as they are known to receive an important contribution from long distance effects in the $\mathrm{J} / \psi$ and $\psi^{\prime}$ mass regions.

\section{The effective hamiltonian for weak decays}

The effective hamiltonian we shall use here for the rare decays $B \rightarrow K^{*} \gamma, B \rightarrow K l \bar{\ell}$ and $B \rightarrow K^{*} \ell \bar{l}$ (with $l \bar{l}=e^{+} e^{-}, \mu^{+} \mu^{-}$or $\left.\nu \bar{v}\right)$ is derived by integrating out the top quark and the $\mathrm{W}$ - and Z-bosons at the same scale $\mu \approx M_{\mathrm{w}}$. An appropriate operator basis for the rare B-decays consists of twelve operators [1-4] and the effective hamiltonian may be written as

$H_{\mathrm{eff}}=-\frac{4 G_{\mathrm{F}}}{\sqrt{2}} V_{\mathrm{tb}} V_{\mathrm{ts}}^{*} \sum_{j=1}^{12} C_{j}(\mu) O_{j}(\mu)$,

where $G_{\mathrm{F}}$ is the Fermi coupling constant, $V_{i j}$ are the Cabibbo-Kobayashi-Maskawa matrix elements, and $C_{j}(\mu)$ are the Wilson coefficients, which are determined by appropriate matching conditions at $\mu=M_{\mathrm{W}}$ and the renormalization group equations to scale down to a lower mass scale, say $\mu=m_{\mathrm{b}}$. Note that only operators of dimension five and six are included here since the higher dimensional operators are suppressed by powers of $m_{\mathrm{b}} / m_{\mathrm{w}}$. Detailed considerations $[3,4]$, however, show that the coefficients of some of the operators and their mixing with the remaining ones are small and the basis may be truncated

$$
H_{\mathrm{eff}}=-\frac{4 G_{\mathrm{F}}}{\sqrt{2}} V_{\mathrm{tb}} V_{\mathrm{ts}}^{*} \sum_{j} C_{j}(\mu) O_{j}(\mu),
$$$$
j=1,2,7,8,9,
$$

where we use the notation of ref. [3]. The operators of interest are given by

$O_{1}\left(m_{\mathrm{b}}\right)=\left(\bar{s} \gamma_{\mu} L b\right)\left(\bar{c} \gamma_{\mu} L c\right)$,
$O_{2}\left(m_{\mathrm{b}}\right)=\left(\bar{s} \gamma_{\mu} L c\right)\left(\bar{c} \gamma_{\mu} L b\right)$

$O_{7}\left(m_{\mathrm{b}}\right)=\frac{e}{16 \pi^{2}} F_{\mu \nu} m_{\mathrm{b}}\left(\bar{s} \sigma_{\mu \nu} R b\right)$,

$O_{8}\left(m_{\mathrm{b}}\right)=\frac{\alpha}{4 \pi}\left(\bar{s} \gamma_{\mu} L b\right)\left(\bar{e} \gamma_{\mu} e\right)$,

$O_{9}\left(m_{\mathrm{b}}\right)=\frac{\alpha}{4 \pi}\left(\bar{s} \gamma_{\mu} L b\right)\left(\bar{e} \gamma_{\mu} \gamma_{5} e\right)$,

with $L=\frac{1}{2}\left(1-\gamma_{5}\right)$ and $R=\frac{1}{2}\left(1+\gamma_{5}\right)$. In eq. (5) we have neglected a term proportional to $m_{\mathrm{s}}$ which is much smaller than the one shown. The Wilson coefficients are given by [1-3]

$$
\begin{aligned}
& C_{1}\left(m_{\mathrm{b}}\right)=-\frac{1}{2}\left(\eta^{-6 / 23}-\eta^{12 / 23}\right) \\
& C_{2}\left(m_{\mathrm{b}}\right)=-\frac{1}{2}\left(\eta^{-6 / 23}+\eta^{12 / 23}\right) \\
& C_{7}\left(m_{\mathrm{b}}\right)=\eta^{16 / 23}\left[C_{7}\left(M_{\mathrm{W}}\right)+\frac{58}{135}\left(\eta^{10 / 23}-1\right)\right. \\
& \left.\quad+\frac{29}{189}\left(\eta^{28 / 23}-1\right)\right] \\
& C_{8}\left(m_{\mathrm{b}}\right)=C_{8}\left(M_{\mathrm{W}}\right) \\
& \quad+\frac{4 \pi}{\alpha_{s}\left(M_{\mathrm{W}}\right)}\left[\frac{4}{33}\left(1-\eta^{-11 / 23}\right)-\frac{8}{87}\left(1-\eta^{-29 / 23}\right)\right]
\end{aligned}
$$

$C_{9}\left(m_{\mathrm{b}}\right)=C_{9}\left(M_{\mathrm{W}}\right)$,

with $\eta=\alpha_{\mathrm{s}}\left(m_{\mathrm{b}}\right) / \alpha_{\mathrm{s}}\left(M_{\mathrm{W}}\right)$. The coefficients at the scale $M_{\mathrm{W}}$ appearing in (10)-(12) are given in refs. $[3,5]$. The various branching ratios of interest depend on the top quark mass, on which we shall comment later; for the numerical estimates below we have chosen the value $m_{\mathrm{t}}=100 \mathrm{GeV}$. Furthermore, we assume $\mu=m_{\mathrm{b}}$ to have the large logarithms of the type $\ln \left(M_{\mathrm{w}}^{2} / m_{\mathrm{b}}^{2}\right)$ included in the coefficients and not in the matrix elements of the operators. Since we eventually use the operators $O_{j}$ to calculate a matrix element for a transition $b \rightarrow s+$ (virtual) photon, we have to take a one loop matrix element of the operators $O_{1}$ and $\mathrm{O}_{2}$. This introduces new logarithms of the type $\ln \left(M_{\mathrm{w}} / m_{\mathrm{b}}\right)$ which are not due to QCD effects and are already included in (11). For details we refer the reader to ref. [3].

\section{Estimates of decay rates}

We briefly introduce the formalism that we employ for calculating the decay matrix elements, and dis- 
cuss the rates for the semileptonic decays $\mathrm{D} \rightarrow \mathrm{K} \ell v_{\ell}$ and $\mathrm{D} \rightarrow \mathrm{K}^{*} \ell v_{\ell}$ in the limit of heavy $\mathrm{c}$ - and s-quarks. Comparing the rates with the corresponding experimental measurements provides an estimate of the errors inherent in neglecting the $1 / m_{\mathrm{s}}$ terms. Then we proceed to calculate the already mentioned rare $\mathrm{B}$ decays in the limit of heavy b-and s-quarks.

\subsection{Hadronic matrix elements}

We start from the bilinear operators involving heavy quarks, which we generically denote as

$$
\bar{h}_{v^{\prime}}^{\prime} \Gamma h_{v} \text {, }
$$

where $\Gamma$ denotes some combination of the Dirac gamma matrices and $v$ and $v^{\prime}$ are the velocities of the heavy quarks of type $h$ and $h^{\prime}$. It is known that all the matrix elements of this type of operators are described in terms of a single universal function $\xi$, the so called Isgur-Wise function [12], which depends only on the product of the two velocities $v \cdot v^{\prime}$. We shall use the trace formalism given in refs. [13,14] and by Falk et al. [11] to evaluate the matrix elements. In this formalism the heavy $0^{-}$mesons $\mathrm{H}$ moving with some velocity $v$ are described by the matrices $\# 1$

$H(v)=\frac{1}{2} \sqrt{m_{\mathrm{H}}} \gamma_{5}(\phi-1)$,

while the matrices for the corresponding $1^{-}$states are given by

$H^{*}(v, \epsilon)=\frac{1}{2} \sqrt{m_{\mathrm{H}}} \notin(\psi-1)$,

where $\epsilon$ is the polarization vector of the $1^{-}$state. The matrix element of the operators (13) are then given by (for example for $1^{-} \rightarrow 1^{-}$transitions)

$$
\begin{aligned}
& \left\langle H^{\prime(*)}\left(v^{\prime}, \epsilon^{\prime}\right)\left|\bar{h}_{v^{\prime}}^{\prime} \Gamma h_{v}\right| H^{(*)}(v, \epsilon)\right\rangle \\
& \quad=\xi\left(v \cdot v^{\prime}\right) \operatorname{Tr}\left\{H^{\prime(*)}\left(v^{\prime}, \epsilon^{\prime}\right) \Gamma H^{(*)}(v, \epsilon)\right\} .
\end{aligned}
$$

In the heavy quark limit, we may relate the rare Bdecays to the semileptonic decays of the D- and Bmesons, since all these decays are described by the same function. We have opted to determine the Isgur-Wise function by using data on the semilep-

\#1 Note that in ref. [13] a different convention for the Dirac matrices is used. Falk et al. (refs. [11,14]) and Luke (ref. [14]) define different matrices which behave correctly under proper Lorentz- and spin symmetry transformations; the additional $\gamma_{5}$ in (14) ensures also the correct parity transformations. tonic decays of the $\mathrm{D}$-meson, namely $\mathrm{D} \rightarrow \mathrm{K} \ell v_{\ell}$ and $\mathrm{D} \rightarrow \mathrm{K}^{*} \ell v_{\ell}$. This is done since we want to use this framework to determine the rates for the rare B-decays, involving the $b \rightarrow s$ transitions, which yield a $K$ or a $\mathrm{K}^{*}$ in the final state.

\subsection{Decay rates for $D \rightarrow K \ell \nu_{\ell}$ and $D \rightarrow K^{*} \ell \nu_{\ell}$}

The amplitudes for the semileptonic decays $\mathrm{D} \rightarrow$ $\mathrm{K} \ell v_{\ell}$ and $\mathrm{D} \rightarrow \mathrm{K}^{*} \ell v_{\ell}$ is

$$
\begin{aligned}
\mathscr{M}^{*} & =\frac{G_{\mathrm{F}}}{\sqrt{2}} V_{\mathrm{cs}}\left\langle\mathrm{K}^{(*)}\left|\bar{s} \gamma_{\mu}\left(1-\gamma_{\mathrm{S}}\right) c\right| \mathrm{D}\right\rangle \\
& \times\left\langle\ell v_{\ell}\left|\bar{\nu} \gamma^{\mu}\left(1-\gamma_{s}\right) \ell\right| 0\right\rangle .
\end{aligned}
$$

The hadronic matrix elements in the limit of heavy c- and s-quarks are given in terms of the Isgur-Wise function by the relations

$$
\begin{aligned}
& \left\langle\mathrm{K}\left(v^{\prime}\right)\left|\bar{s} \gamma_{\mu}\left(1-\gamma_{5}\right) c\right| \mathrm{D}(v)\right\rangle \\
& =\sqrt{m_{\mathrm{K}} m_{\mathrm{D}}} \xi\left(v \cdot v^{\prime}\right)\left(v_{\mu}+v_{\mu}^{\prime}\right), \\
& \left\langle\mathrm{K}\left(v^{\prime}, \boldsymbol{\epsilon}\right)\left|\bar{s} \gamma_{\mu}\left(1-\gamma_{5}\right) c\right| \mathrm{D}(v)\right\rangle=\sqrt{m_{\mathrm{K}} m_{\mathrm{D}}} \xi\left(v \cdot v^{\prime}\right) \\
& \quad \times\left[\left(1+v \cdot v^{\prime}\right) \epsilon_{\mu}-v_{\mu}^{\prime}(\boldsymbol{\epsilon} \cdot v)+\mathbf{i} \boldsymbol{\epsilon}_{\mu \alpha \beta \gamma} v^{\alpha} v^{\prime \beta} \epsilon^{\nu}\right] .
\end{aligned}
$$

In order to obtain the total rates we have to specify a parametrization of the Isgur-Wise function. (This is equivalent to assuming a parametrization of the usual form factors in the decays.) We shall use two different parametrizations; the first one is a monopole form,

$\xi\left(v \cdot v^{\prime}\right)=\frac{w_{0}^{2}}{w_{0}^{2}-2+2 v \cdot v^{\prime}}$,

and the second one is encountered in models employing wave functions to calculate the decay form factors:

$\xi\left(v \cdot v^{\prime}\right)=\exp \left[\beta\left(1-v \cdot v^{\prime}\right)\right]$.

We use the data on $\mathrm{D} \rightarrow \mathrm{K} \ell v_{\ell}$ to fit $w_{0}$ in (20) and $\beta$ in $(21)$. The best fit is obtained for $w_{0} \approx 1.80$ and $\beta \approx 0.5$. Table 1 shows the results for some measured quantities for the two cases. The experimental values for $\Gamma$ and $\Gamma^{*}$ are taken from the Particle Data Group [16], while the values for $\Gamma_{\mathrm{L}}^{*} / \Gamma_{\mathrm{T}}^{*}$ and $\Gamma^{*} / \Gamma$ are the weighted averages of the recent measurements of these quantities by the E691 [17] and MARK III [18] Collaborations. A couple of remarks are in order at this point. It is clear that from the semileptonic rates 
Table 1

Comparison of experiment and theory for the decay rates $\mathrm{D} \rightarrow \mathrm{K} \ell v_{\ell}$ and $\mathrm{D} \rightarrow \mathrm{K}^{(*)} \ell v_{\mathrm{\ell}}$. Here $\Gamma_{\mathrm{L}}^{*}\left(\Gamma_{\mathrm{T}}^{*}\right)$ denotes the decay rate for the longitudinal (transverse) polarisation mode of the $\mathrm{K}^{*}$.

\begin{tabular}{lcccc}
\hline & $\Gamma(\mathrm{GeV})$ & $\Gamma^{*}(\mathrm{GeV})$ & $\Gamma_{\mathrm{L}}^{*} / \Gamma_{\mathrm{T}}^{*}$ & $\Gamma^{*} / \Gamma$ \\
\hline eq. $(20), w_{0}=1.80$ & $5.3 \times 10^{-14}$ & $6.3 \times 10^{-14}$ & 1.17 & 1.19 \\
eq. $(21), \beta=0.50$ & $5.3 \times 10^{-14}$ & $6.4 \times 10^{-14}$ & 1.21 & 1.21 \\
experiment & $(5.30 \pm 0.62) \times 10^{-14}$ & $(2.36 \pm 0.43) \times 10^{-14}$ & $1.15 \pm 0.7$ & $0.75 \pm 0.30$ \\
\hline
\end{tabular}

alone the two parametrizations of the Isgur-Wise function are hardly distinguishable. However, experimental analyses of the D-meson decay form factors are in good agreement with a monopole form [17]. The calculations in the static limit presented here predict a factor 2 larger decay width for the $\mathrm{D} \rightarrow \mathrm{K}^{*} \ell v_{\ell}$ mode, though the ratio $\Gamma_{\mathrm{L}}^{*} / \Gamma_{\mathrm{T}}^{*}$ predicted for this mode is in good agreement with the data. This just reflects that the s-quark is not particularly heavy and significant recoil corrections are present. However, if a factor 2 is a typical uncertainty due to the neglect of $1 / m_{\mathrm{s}}$ terms in the $\mathrm{c} \rightarrow \mathrm{s}$ transitions, then probably a comparable precision should also be anticipated in the $b \rightarrow s$ transitions. The predictions that we are about to make for the exclusive decay rates in rare B-decays should then hold within this accuracy.

\subsection{Decay rate for $B \rightarrow K^{*}$}

For the decay $\mathrm{B} \rightarrow \mathrm{K}^{*} \gamma$ only the operator $O_{7}$ from the list given in section 2 contributes:

$$
\begin{aligned}
& \left\langle\mathrm{K}^{*} \gamma\left|H_{\mathrm{eff}}\right| \mathrm{B}\right\rangle \\
& =-\frac{4 G_{\mathrm{F}}}{\sqrt{2}} V_{\mathrm{tb}} V_{\mathrm{ts}}^{*} C_{7}\left(m_{\mathrm{b}}\right)\left\langle\mathrm{K}^{*} \gamma\left|O_{7}\left(m_{\mathrm{b}}\right)\right| \mathrm{B}\right\rangle .
\end{aligned}
$$

The hadronic matrix element needed for $(22)$ is now evaluated in the heavy quark limit for the $b$ - and $s$-quarks. It is thus given in terms of the Isgur-Wise function, as for the already discussed case of D-semileptonic decays. In the heavy quark limit the operator $\mathrm{O}_{7}$ simplifies. The derivative from the field strength gives a factor $q=m_{\mathrm{B}} v-m_{\mathrm{K}} v^{\prime}$ and, since in the heavy quark limit $\phi^{\prime} s=s$ and $\phi b=b$, we have

$$
\begin{gathered}
\bar{s} \sigma^{\mu \nu}\left(1+\gamma_{5}\right) b q^{\nu}=-m_{\mathrm{B}} \bar{s} \gamma_{\mu}\left(1-\gamma_{5}\right) b \\
-m_{\mathrm{B}} \bar{s} \gamma_{\mu}\left(1+\gamma_{5}\right) b+q_{\mu} \bar{s}\left(1+\gamma_{5}\right) b .
\end{gathered}
$$

$$
\begin{aligned}
& \left\langle\mathrm{K}^{*}\left(v^{\prime}, \epsilon\right)\left|\bar{s}_{\mu}\left(1 \mp \gamma_{5}\right) b\right| \mathrm{B}(v)\right\rangle \\
& =\sqrt{m_{\mathrm{K}^{*}} m_{\mathrm{B}}} \xi\left(v \cdot v^{\prime}\right) \\
& \quad \times\left[\left(1+v \cdot v^{\prime}\right) \epsilon_{\mu}-v_{\mu}^{\prime}(\epsilon \cdot v) \pm \mathrm{i} \epsilon_{\mu \alpha \beta \gamma} v^{\alpha} v^{\prime \beta} \epsilon^{\gamma}\right], \\
& \left\langle\mathrm{K}^{*}\left(v^{\prime}, \epsilon\right)\left|\bar{s}\left(1+\gamma_{5}\right) b\right| \mathrm{B}(v)\right\rangle \\
& =\sqrt{m_{\mathrm{K}^{*}} m_{\mathrm{B}}} \xi\left(v \cdot v^{\prime}\right)(\epsilon \cdot v) .
\end{aligned}
$$

This then leads to the decay rate

$$
\begin{aligned}
& \Gamma\left(\mathrm{B} \rightarrow \mathrm{K}^{*} \gamma\right) \\
& \quad=\frac{1}{16 \pi^{4}} G_{\mathrm{F}}^{2} \alpha\left|\xi\left(v \cdot v^{\prime}\right)\right|^{2}\left|V_{\mathrm{tb}}\right|^{2}\left|V_{\mathrm{ts}}\right|^{2}\left|C_{7}\left(m_{\mathrm{B}}\right)\right|^{2} \\
& \quad \times(k \cdot v)^{3} m_{\mathrm{B}}^{2} \frac{m_{\mathrm{K}^{*}}}{m_{\mathrm{B}}}\left(1+\frac{m_{\mathrm{B}}}{m_{\mathrm{K}^{*}}}\right)^{2},
\end{aligned}
$$

where the Isgur-Wise function is evaluated at the point

$v \cdot v^{\prime}=\frac{1}{2 m_{\mathrm{B}} m_{\mathrm{K}^{*}}}\left(m_{\mathrm{B}}^{2}+m_{\mathrm{K}^{*}}^{2}\right) \approx 3.04$.

This value is far outside the kinematic range of the semileptonic D-decays; the maximum value of $v \cdot v^{\prime}$ in $\mathrm{D} \rightarrow \mathrm{Ke} v$ and $\mathrm{D} \rightarrow \mathrm{K}^{*} \mathrm{e} v$ is $v \cdot v^{\prime} \approx 2.01$ and $v \cdot v^{\prime} \approx 1.29$, respectively. We shall continue employing the parametrizations of the Isgur-Wise function given in the last section also for this large value of $v \cdot v^{\prime}$. Eq. (26) may be compared to the inclusive rate $B \rightarrow X_{s} \gamma$, which is usually taken to be the QCD improved quark decay rate for $b \rightarrow s \gamma$. In this approximation, the expression for the inclusive rate can be expressed as $[1,2]$

$$
\begin{aligned}
& \Gamma\left(\mathrm{B} \rightarrow \mathrm{X}_{\mathrm{s}} \gamma\right) \\
& \quad=\frac{G_{\mathrm{F}}^{2}}{4 \pi^{4}} \alpha\left|V_{\mathrm{tb}}\right|^{2}\left|V_{\mathrm{ts}}\right|^{2}\left|C_{7}\left(m_{\mathrm{B}}\right)\right|^{2}(k \cdot v)^{3} m_{\mathrm{B}}^{2} .
\end{aligned}
$$

Defining the exclusive to inclusive decay rate ratio

The hadronic matrix elements are 
Table 2

Decay rates and branching ratios for the decay $B \rightarrow K^{*} \gamma$, for $m_{\mathbf{t}}=100 \mathrm{GeV}$.

\begin{tabular}{llll}
\hline & $\Gamma\left(\mathrm{B} \rightarrow \mathrm{K}^{*} \gamma\right)(\mathrm{GeV})$ & $\mathrm{BR}\left(\mathrm{B} \rightarrow \mathrm{K}^{*} \gamma\right)$ & $R\left(\mathrm{~B} \rightarrow \mathrm{K}^{*} \gamma\right)(\%)$ \\
\hline eq. $(20), w_{0}=1.80$ & $3.8 \times 10^{-17}$ & $6.8 \times 10^{-5}$ & 18 \\
eq. $(21), \beta=0.50$ & $6.1 \times 10^{-17}$ & $1.1 \times 10^{-4}$ & 28 \\
\hline
\end{tabular}

$R\left(\mathrm{~B} \rightarrow \mathrm{K}^{*} \gamma\right) \equiv \frac{\Gamma\left(\mathrm{B} \rightarrow \mathrm{K}^{*} \gamma\right)}{\Gamma\left(\mathrm{B} \rightarrow \mathrm{X}_{\mathrm{s}} \gamma\right)}$

we can express this ratio in a compact form:

$R\left(\mathrm{~B} \rightarrow \mathrm{K}^{*} \gamma\right)=\frac{m_{\mathrm{K}^{*}}}{4 m_{\mathrm{B}}}\left|\xi\left(v \cdot v^{\prime}\right)\right|^{2}\left(1+\frac{m_{\mathrm{B}}}{m_{\mathrm{K}^{*}}}\right)^{2}$.

Table 2 contains the predictions for the total rate, the branching rate, the branching fractions and the ratio $R\left(\mathrm{~B} \rightarrow \mathrm{K}^{*} \gamma\right)$ for the two assumed parametrizations of the Isgur-Wise function. We compare the branching ration $\mathrm{BR}\left(\mathrm{B} \rightarrow \mathrm{K}^{*} \gamma\right)$ and the ratio $R\left(\mathrm{~B} \rightarrow \mathrm{K}^{*} \gamma\right)$ obtained here with calculations of the corresponding quantities in the literature. In ref. [6], an effective hamiltonian approach based on QCD and an experimentally constrained wave function model for the Bmeson were used to estimate $B R\left(B \rightarrow K^{*} \gamma\right)=(3-$ $6) \times 10^{-5}$ and $R\left(\mathrm{~B} \rightarrow \mathrm{K}^{*} \gamma\right)=(10-18) \%$. The authors of ref. [8] obtained $R\left(\mathrm{~B} \rightarrow \mathrm{K}^{*} \gamma\right)=15 \%$, based on QCD sum rules supplemented by a pole model. These numbers are in good agreement with the present estimated based on the monopole parametrization, whereas the ones corresponding to the exponential form are significantly higher. While we cannot argue persuasively based on the consistency of these model calculations, yet a preference for the monopole parametrization of the Isgur-Wise function is suggested by these comparisons as well.

\subsection{Decay rates for $B \rightarrow K \ell^{+} \ell^{-}, B \rightarrow K^{*} \ell^{+} \ell^{-}$}

In these decays all the five operators given above are relevant. The contribution of $O_{1}$ and $O_{2}$ is via a one loop diagram which renders the invariant mass spectrum of the lepton pair nontrivial. Their contribution may be written as

$$
\begin{aligned}
\tilde{O}_{1} & =3 \tilde{O}_{2} \\
& =\left[\bar{s} \gamma_{\mu}\left(1+\gamma_{5}\right) b\right]\left(I_{\gamma_{\mu}} l\right) g\left(m_{\ell \ell}^{2}\right),
\end{aligned}
$$

where $m_{\ell \ell}^{2}$ denotes the invariant mass of the lepton pair and the function $g(y)$ is given by

$$
\begin{gathered}
g(y)=-\left[\frac{4}{9} \ln \left(\frac{m_{\mathrm{c}}^{2}}{m_{\mathrm{b}}^{2}}\right)-\frac{8}{27}-\frac{16}{9} \frac{m_{\mathrm{c}}^{2}}{y}\right. \\
\left.+\frac{2}{9} \sqrt{1-\frac{4 m_{\mathrm{c}}^{2}}{y} \mid}\left(2+\frac{4 m_{\mathrm{c}}^{2}}{y}\right) G(y)\right],
\end{gathered}
$$

with

$$
\begin{aligned}
& G(y)=\ln \left|\frac{1+\sqrt{1-4 m_{\mathrm{c}}^{2} / y}}{1-\sqrt{1-4 m_{\mathrm{c}}^{2} / y}}\right|+\mathrm{i} \pi \\
& \text { for } y>4 m_{\mathrm{c}}^{2}, \\
& G(y)=2 \arctan \left(\frac{1}{\sqrt{1-4 m_{\mathrm{c}}^{2} / y}}\right) \\
& \text { for } y<4 m_{\mathrm{c}}^{2} .
\end{aligned}
$$

Note that $g(y)$ is renormalization scheme dependent; this dependence is cancelled by that of the Wilson coefficient $C_{8}$ [5]. The operator $O_{7}$ contributes via an additional virtual photon, while the contributions of the remaining two operators are trivial.

The hadronic matrix elements are evaluated as before in terms of the Isgur-Wise function and are given by

$$
\begin{aligned}
& \left\langle\mathrm{K}\left(v^{\prime}\right)\left|\bar{s} \gamma_{\mu}\left(1 \mp \gamma_{5}\right) b\right| \mathrm{B}(v)\right\rangle \\
& =\sqrt{m_{\mathrm{K}} m_{\mathrm{B}}} \xi\left(v \cdot v^{\prime}\right)\left(v_{\mu}+v_{\mu}^{\prime}\right), \\
& \left\langle\mathrm{K}\left(v^{\prime}\right)\left|\bar{s}\left(1+\gamma_{5}\right) b\right| \mathrm{B}(v)\right\rangle \\
& =\sqrt{m_{\mathrm{K}} m_{\mathrm{B}}} \xi\left(v \cdot v^{\prime}\right)\left(1+v \cdot v^{\prime}\right), \\
& \left\langle\mathrm{K}^{*}\left(v^{\prime}, \epsilon\right)\left|\bar{s} \gamma_{\mu}\left(1 \mp \gamma_{5}\right) b\right| \mathrm{B}(v)\right\rangle \\
& =\sqrt{m_{\mathrm{K}} m_{\mathrm{B}}} \xi\left(v \cdot v^{\prime}\right) \\
& \quad \times\left[\left(1+v \cdot v^{\prime}\right) \epsilon_{\mu}-v_{\mu}^{\prime}(\epsilon \cdot v) \pm \mathrm{i} \epsilon_{\mu \alpha \beta \gamma} v^{\alpha} v^{\prime} \epsilon^{\nu}\right],
\end{aligned}
$$




$$
\begin{gathered}
\left\langle\mathrm{K}^{*}\left(v^{\prime}, \epsilon\right)\left|\bar{s}\left(1+\gamma_{\mathrm{s}}\right) b\right| \mathrm{B}(v)\right\rangle \\
=\sqrt{m_{\mathrm{K}^{*}} m_{\mathrm{B}}} \xi\left(v \cdot v^{\prime}\right)(\epsilon \cdot v) .
\end{gathered}
$$

Incorporating this in the effective hamiltonian we derive the following invariant dilepton mass differential rates:

$$
\begin{aligned}
& \frac{\mathrm{d} \Gamma}{\mathrm{d} m_{\mathrm{l \ell}}^{2}}\left(\mathrm{~B} \rightarrow \mathrm{K} \ell^{+} \ell^{-}\right)=\frac{G_{\mathrm{F}}^{2} m_{\mathrm{B}}^{3}}{(2 \pi)^{3}}\left|V_{\mathrm{tb}} V_{\mathrm{ts}}^{*}\right|^{2} \frac{1}{48 r}\left(\frac{\alpha}{4 \pi}\right)^{2} \\
& \quad \times\left(Q_{+} Q_{-}\right)^{3 / 2}(1+r)^{2} \xi^{2}\left(v \cdot v^{\prime}\right) \\
& \quad \times\left(\left|C_{8 \mathrm{eff}}\left(m_{\mathrm{ll}}^{2}, m_{\mathrm{b}}\right)+\frac{2}{1+r} C_{7}\left(m_{\mathrm{B}}\right)\right|^{2}\right. \\
& \left.\quad+\left|C_{9}\left(m_{\mathrm{B}}\right)\right|^{2}\right), \\
& \frac{\mathrm{d} \Gamma^{*}}{\mathrm{~d} m_{\mathrm{ll}}^{2}}\left(\mathrm{~B} \rightarrow \mathrm{K}^{*} \ell^{+} \ell^{-}\right)=\frac{G_{\mathrm{F}}^{2} m_{\mathrm{B}}^{3}}{(2 \pi)^{3}}\left|V_{\mathrm{tb}} V_{\mathrm{ts}}^{*}\right|^{2} \frac{1}{48 r}\left(\frac{\alpha}{4 \pi}\right)^{2} \\
& \quad \times Q_{+} \sqrt{Q_{+} Q_{-}} \xi^{2}\left(v \cdot v^{\prime}\right) \\
& \quad \times\left[\left[\left|C_{8 \mathrm{eff}}\left(m_{\mathrm{ll}}^{2}, m_{\mathrm{b}}\right)\right|^{2}+\left|C_{9}\left(m_{\mathrm{B}}\right)\right|^{2}\right]\right. \\
& \quad \times\left[4 \hat{x}^{2}\left(1+r^{2}-\hat{x}^{2}\right)+Q_{+}(1-r)^{2}\right] \\
& \quad+4\left|C_{7}\left(m_{\mathrm{b}}\right)\right|^{2}\left(\frac{4}{\hat{x}^{2}}\left[\left(1-r^{2}\right)^{2}-\hat{x}^{2}\right]+Q_{+}\right) \\
& \left.\quad+16 \mathrm{Re}\left[C_{7}\left(m_{\mathrm{b}}\right) C_{8} \mathrm{eff}\left(m_{\mathrm{l \ell}}^{2}, m_{\mathrm{b}}\right)\right]\left(1-r^{2}-\hat{x}^{2}\right)\right],
\end{aligned}
$$

where we have used the abbreviations

$$
\begin{aligned}
& C_{8 \mathrm{eff}}\left(m_{\ell \ell}^{2}, m_{\mathrm{B}}\right) \\
& \quad=C_{8}\left(m_{\mathrm{B}}\right)+\left[3 C_{1}\left(m_{\mathrm{B}}\right)+C_{2}\left(m_{\mathrm{B}}\right)\right] g\left(m_{\ell \ell}^{2}\right), \\
& \hat{x}^{2}=\frac{m_{\ell \ell}^{2}}{m_{\mathrm{B}}^{2}}, \quad r=\frac{m_{\mathrm{K}}(*)}{m_{\mathrm{B}}}, \\
& Q_{ \pm}=(1 \pm r)^{2}-\hat{x}^{2} \\
& v \cdot v^{\prime}=\frac{1}{2 r}\left(1+r^{2}-\hat{x}^{2}\right) .
\end{aligned}
$$

Integrating the differential distributions given above, the decay rates are given in tables 3 and 4 for the two parametrizations of the Isgur-Wise function. Note that the differential rate for $\mathrm{B} \rightarrow \mathrm{K}^{*} \mathrm{e}^{+} \mathrm{e}^{-}$becomes singular for $\hat{x} \rightarrow 0$ and thus the total rate will depend logarithmically on the kinematic lower bound, namely $4 m_{\ell}^{2} / m_{\mathrm{B}}^{2}$. This is the only point in our analysis where lepton mass effects become important. The

\begin{tabular}{|c|c|c|c|c|c|c|}
\hline & $\begin{array}{l}\Gamma\left(\mathrm{B} \rightarrow \mathrm{K}^{*} \mathrm{e}^{+} \mathrm{e}^{-}\right) \\
(\mathrm{GeV})\end{array}$ & $\mathrm{BR}\left(\mathrm{B} \rightarrow \mathrm{K}^{*} \mathrm{e}^{+} \mathrm{e}^{-}\right)$ & $\begin{array}{l}R\left(\mathrm{~B} \rightarrow \mathrm{K}^{*} \mathrm{e}^{+} \mathrm{e}^{-}\right) \\
(\%)\end{array}$ & $\begin{array}{l}\Gamma\left(\mathrm{B} \rightarrow \mathrm{K}^{*} \mu^{+} \mu^{-}\right) \\
(\mathrm{GeV})\end{array}$ & $\mathrm{BR}\left(\mathrm{B} \rightarrow \mathrm{K}^{*} \boldsymbol{\mu}^{+} \boldsymbol{\mu}^{-}\right)$ & $\begin{array}{l}R\left(\mathrm{~B} \rightarrow \mathrm{K}^{*} \mu^{+} \mu^{-}\right) \\
(\%)\end{array}$ \\
\hline eq. $(20), w_{0}=1.80$ & $1.3 \times 10^{-18}$ & $2.3 \times 10^{-6}$ & 35 & $6.7 \times 10^{-19}$ & $1.2 \times 10^{-6}$ & 35 \\
\hline eq. $(21), \beta=0.50$ & $9.6 \times 10^{-19}$ & $1.7 \times 10^{-6}$ & 27 & $5.7 \times 10^{-19}$ & $1.0 \times 10^{-6}$ & 26 \\
\hline
\end{tabular}
rate for $\mathrm{B} \rightarrow$ Kee does not become singular in this limit, and thus the difference between the rates for $\mathrm{B} \rightarrow \mathrm{Kee}$ and $B \rightarrow K \mu \mu$ is negligible.

We also give in tables 3 and 4 the ratio $R$, defined analogously to the ratio $R\left(\mathrm{~B} \rightarrow \mathrm{K}^{*} \gamma\right)$ previously, for the indicated exclusive decays, with the inclusive rate for $B \rightarrow X_{s}$ ee and $B \rightarrow X_{s} \mu \mu$ taken from ref. [3]:

$$
\begin{aligned}
& \Gamma\left(\mathrm{B} \rightarrow \mathrm{X}_{\mathrm{s}} \mathrm{ee}\right)=3.63 \times 10^{-18} \mathrm{GeV}, \\
& \Gamma\left(\mathrm{B} \rightarrow \mathrm{X}_{\mathrm{s}} \mu \mu\right)=1.94 \times 10^{-18} \mathrm{GeV} .
\end{aligned}
$$

The numbers in table 3 and 4 are in agreement with

Table 3

Decay rates, branching fractions and the ratios $R$ for the decays $\mathrm{B} \rightarrow \mathrm{K} \ell^{+} \ell^{-}$with $\ell=\mathrm{e}, \mu\left(m_{\mathrm{t}}=100 \mathrm{GeV}\right)$.

\begin{tabular}{lllll}
\hline & $\Gamma\left(\mathrm{B} \rightarrow \mathrm{K} \ell^{+} \ell^{-}\right)(\mathrm{GeV})$ & $\mathrm{BR}\left(\mathrm{B} \rightarrow \mathrm{K} \ell^{+} \ell^{-}\right)$ & $R\left(\mathrm{~B} \rightarrow \mathrm{Ke}^{+} \mathrm{e}^{-}\right)(\%)$ & $R\left(\mathrm{~B} \rightarrow \mathrm{K} \mu^{+} \mu^{-}\right)(\%)$ \\
\hline eq. $(20), w_{0}=1.80$ & $1.4 \times 10^{-19}$ & $2.5 \times 10^{-7}$ & 4 & 7 \\
eq. (21), $\beta=0.50$ & $6.3 \times 10^{-20}$ & $1.1 \times 10^{-7}$ & 2 & 3 \\
\hline
\end{tabular}

Table 4

Decay rates, branching fractions and the ratios $R$ for the decays $\mathrm{B} \rightarrow \mathrm{K}^{*} \mathrm{e}^{+} \mathrm{e}^{-}$and $\mathrm{B} \rightarrow \mathrm{K}^{*} \mu^{+} \mu^{-}\left(m_{\mathrm{t}}=100 \mathrm{GeV}\right)$. 
the corresponding estimates in refs. [8,9]. The importance of including the long distance contributions from the decays $B \rightarrow J / \psi\left(K, K^{*}\right) \rightarrow \ell^{+} \ell^{-}\left(K, K^{*}\right)$, in particular the resulting interference pattern in the invariant dilepton mass distributions, is well noted in the literature. We shall present these distributions in a forthcoming publication.

\subsection{Decay rates for $B \rightarrow K \nu \bar{\nu}$ and $B \rightarrow K^{*} \nu \bar{\nu}$}

These processes are experimentally very difficult to measure, except probably in a high luminosity LEP environment, involving the decay $Z^{0} \rightarrow b \bar{b}$, where such events have a nice tag of large missing energy and momentum in a b-quark jet with no charged lepton. From the theoretical point of view, on the other hand, these decays are much simpler to deal with, since the operator basis given in section 2 reduces to only one operator, which is the difference of $O_{8}$ and $O_{9}$. Thus, a left handed current for the neutrinos appears which couples pointlike to the left handed current of the $b \rightarrow s$ transition.

The Wilson coefficient of this operator is $D_{9}$ [19]

$D_{9}\left(M_{\mathrm{W}}\right)=E(x)$,

$E(x)=\frac{1}{\sin ^{2} \theta_{\mathrm{w}}}\left(\frac{1}{8} x+\frac{3}{8} \frac{x(x-2)}{(x-1)^{2}} \ln x+\frac{3}{8} \frac{x}{x-1}\right)$,

where $x=m_{\mathrm{t}}^{2} / M_{\mathrm{W}}^{2} . D_{9}$, like $C_{9}$, is not affected by QCD scaling corrections. This gives

$H_{\mathrm{eff}}=-\frac{8 G_{\mathrm{F}}}{\sqrt{2}} V_{\mathrm{tb}} V_{\mathrm{ts}}^{*} D_{9}\left(M_{\mathrm{w}}\right) \frac{\alpha}{4 \pi}\left(\bar{s} \gamma_{\mu} L b\right)\left(\bar{\nu} \gamma_{\mu} L \nu\right)$.

The hadronic matrix elements are given in the previous section and the differential distribution in the missing invariant mass, for three neutrino generations, can be written as

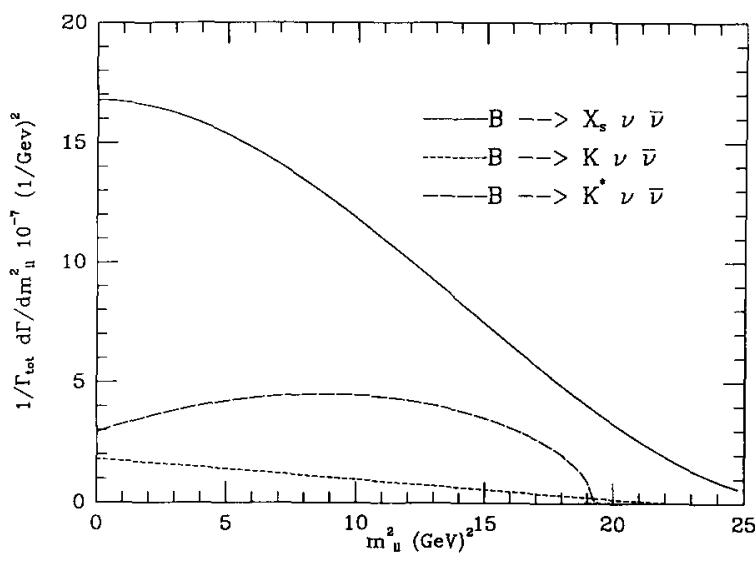

Fig. 1. Differential missing invariant mass distributions in rare B-decays: $\mathrm{B} \rightarrow \mathrm{X}_{\mathrm{s}} \bar{v} \bar{v}$ (solid), $\mathrm{B} \rightarrow \mathrm{K} v \bar{v}$ (short dashed) and $\mathrm{B} \rightarrow$ $K^{*} \bar{w}$ (long dashed).

$$
\begin{aligned}
& \frac{\mathrm{d} \Gamma}{\mathrm{d} m_{v v}^{2}}(\mathrm{~B} \rightarrow \mathrm{K} v \bar{v})=\frac{G_{\mathrm{F}}^{2} m_{\mathrm{B}}^{3}}{(2 \pi)^{3}}\left|V_{\mathrm{tb}} V_{\mathrm{ts}}^{*}\right|^{2}\left(\frac{\alpha}{4 \pi}\right)^{2} \frac{1}{8 r} \\
& \quad \times\left(Q_{+} Q_{-}\right)^{3 / 2}(1+r)^{2} \xi^{2}\left(v \cdot v^{\prime}\right)\left|D_{9}\left(M_{\mathrm{w}}\right)\right|^{2}, \\
& \frac{\mathrm{d} \Gamma^{*}}{\mathrm{~d} m_{l \ell}^{2}}\left(\mathrm{~B} \rightarrow \mathrm{K}^{*} v \bar{v}\right)=\frac{G_{\mathrm{F}}^{2} m_{\mathrm{B}}^{3}}{(2 \pi)^{3}}\left|V_{\mathrm{tb}} V_{\mathrm{ts}}^{*}\right|^{2}\left(\frac{\alpha}{4 \pi}\right)^{2} \frac{1}{8 r} \\
& \quad \times Q_{+} \sqrt{Q_{+} Q_{-}} \xi^{2}\left(v \cdot v^{\prime}\right)\left|D_{9}\left(M_{\mathrm{W}}\right)\right|^{2} \\
& \quad \times\left[4 \hat{m}^{2}\left(1+r^{2}-\hat{m}^{2}\right)+Q_{+}\left(1-r^{2}\right)\right] .
\end{aligned}
$$

In fig. 1 we plot the missing invariant mass spectra using eq. (20) with $w_{0}=1.80$ as a parametrization of the Isgur-Wise function. For comparison we also show the inclusive spectrum for $B \rightarrow X_{s} v \bar{v}$ derived from the expressions in ref. [3].

Integrating the differential distributions yields the rates given in table 5, for the two parametrizations of the Isgur-Wise function. Again, we give the total rate, the branching fraction and the ratio $R$ for the decay

Table 5

Decay rates, branching fractions and the ratios $R$ for the decays $B \rightarrow K v \bar{v}$ and $\mathbf{B} \rightarrow \mathrm{K}^{*} \mathbf{v} \bar{v}\left(m_{\mathrm{t}}=100 \mathrm{GeV}\right)$.

\begin{tabular}{lllllll}
\hline & $\begin{array}{l}\Gamma(\mathrm{B} \rightarrow \mathrm{K} v \bar{v}) \\
(\mathrm{GeV})\end{array}$ & $\mathrm{BR}(\mathrm{B} \rightarrow \mathrm{K} v \bar{v})$ & $\begin{array}{l}R(\mathrm{~B} \rightarrow \mathrm{K} v \bar{v}) \\
(\%)\end{array}$ & $\begin{array}{l}\Gamma\left(\mathrm{B} \rightarrow \mathrm{K}^{*} v \bar{v}\right) \\
(\mathrm{GeV})\end{array}$ & $\begin{array}{l}\mathrm{BR}\left(\mathrm{B} \rightarrow \mathrm{K}^{*} v \bar{v}\right) \\
R(\mathrm{~B} \rightarrow \mathrm{K} * \bar{v}) \\
(\%)\end{array}$ \\
\hline eq. (20), $w_{0}=1.80$ & $1.3 \times 10^{-18}$ & $2.3 \times 10^{-6}$ & 8 & $4.7 \times 10^{-18}$ & $8.2 \times 10^{-6}$ & 30 \\
eq. (21), $\beta=0.50$ & $5.8 \times 10^{-19}$ & $1.0 \times 10^{-6}$ & 4 & $4.3 \times 10^{-18}$ & $7.5 \times 10^{-6}$ & 28 \\
\hline
\end{tabular}


$B \rightarrow\left(K, K^{*}\right) v \bar{v}$ summed over three generations. The inclusive rate for $\mathrm{B} \rightarrow \mathrm{X}_{\mathrm{s}} v \bar{v}$ has been derived in ref. [3] and is (for $m_{1}=100 \mathrm{GeV}$ )

$$
\Gamma\left(\mathrm{B} \rightarrow \mathrm{X}_{\mathrm{s}} \vee \bar{v}\right)=1.5 \times 10^{-17} \mathrm{GeV} \text {. }
$$

\section{Discussion and conclusion}

We remark that the branching ratios given in the tables above, which are calculated for $m_{\mathrm{t}}=100 \mathrm{GeV}$, are enhanced by approximately a factor of $2.0,2.5$, $3.0,4.0$, for the decays $\mathrm{b} \rightarrow \mathrm{s} \ell^{+} \ell^{-}, \mathrm{B} \rightarrow \mathrm{K}^{*} \ell^{+} \ell^{-}$, $\mathrm{B} \rightarrow \mathrm{K}^{+} \ell^{-}$, and $\mathrm{b} \rightarrow \mathrm{s} v \bar{v}$, respectively, for $m_{\mathbf{t}}=200$ $\mathrm{GeV}$. The exclusive to inclusive decay rate ratios, $R$, are however, very stable against the variation of $m_{1}$.

We conclude by pointing out that, as opposed to the decays $\mathrm{B} \rightarrow \mathrm{K}^{*} \gamma$ and $\mathrm{B} \rightarrow \mathrm{K} \ell^{+} \ell^{-}$which constitute only a small fraction of the corresponding inclusive rates, the exclusive decays $B \rightarrow K^{*}$ ee and $B \rightarrow K^{*} \mu \mu$ are expected to be a large fraction of the respective inclusive rates. This is mainly due to the fact that the differential rates, both for the inclusive- and the exclusive decays, $\mathrm{B} \rightarrow \mathrm{K}^{*} \mathrm{ee}, \mathrm{B} \rightarrow \mathrm{K}^{*} \mu \mu$, have a $1 / \hat{m}$ behaviour ( $\hat{m}$ being the invariant mass of the lepton pair), while the rates for $B \rightarrow$ Kee and $B \rightarrow K \mu \mu$ remain finite for $\hat{m} \rightarrow 0$. The results obtained in the limit for a heavy s-quark are consistent with other QCD based estimates within a factor 2 , and within this margin with the experimental data on D-meson decays, wherever such comparisons are possible. This gives hope that also in the decays involving the heavy to light transitions, $\mathrm{B} \rightarrow\left(\mathrm{K}, \mathrm{K}^{*}\right)+\left(\gamma, \ell^{+} \ell^{-}, v \bar{v}\right)$, a similar accuracy may prevail. It would be nice to check the stability of the leading contributions against the $1 / m_{\mathrm{s}}$ corrections in both the semileptonic decays of the D-meson and the rare B-decays considered here.

\section{Acknowledgement}

We acknowledge useful discussions with Christoph Greub and Nello Paver.

\section{References}

[1] M. Shifman, A.I.Vainshtein and V. Zakharov, Phys. Rev. D

$$
18 \text { (1978) 2583; }
$$

54
B. Campbell and P. O'Donnell, Phys. Rev. D 25 (1982) 1989;

S. Bertolini, F. Borzumati and A. Masiero, Phys. Rev. Lett. 59 (1987) 180;

N. Deshpande et al., Phys. Rev. Lett. 59 (1987) 183;

R. Grigjanis et al., Phys. Lett. B 213 (1988) 355.

[2] P. O'Donnell, Phys. Lett. B 175 (1986) 369;

N. Deshpande and J. Trampetic, Phys. Rev. Lett. 60 (1988) 2583;

W. Jaus and D. Wyler, Phys. Rev. D 41 (1990) 3405; S. Bertolini et al., MPI München report MPI-PAE/PTh43/ 90(1990).

[3] B. Grinstein, M.J. Savage and M.B. Wise, Nucl. Phys. B 319 (1989) 271.

[4] G. Carla, G. Ricciardi and A. Viceré, Universita di Pisa Report IFUP-TH 40/90(1990).

[5] B. Grinstein, R. Springer and M.B. Wise, Nucl. Phys. B 339 (1990) 269.

[6] A. Ali and C. Greub, DESY report 90/102(1990); DESY report 90/164(1990).

[7] T. Altomari, Phys. Rev. D 37 (1988) 677.

[8] C. Dominguez, N. Paver and Riazuddin, Phys. Lett. B 214 (1988) 459; Z. Phys. C 48 (1990) 55.

[9] N. Deshpande, J. Trampetic and K. Panose, Phys. Lett. B 214 (1988) 467;

C.S. Lim, T. Morozumi and A.I. Sanda, Phys. Lett. B 218 (1989) 343.

[10] N. Deshpande, Oregon Institute of Theoretical Physics report OITS 413 (1989).

[11] H. Politzer and M. Wise, Phys. Lett. B 206 (1988) 681; Phys. Lett. B 208 ( 1988 ) 504;

M. Voloshin and M. Shifman, Sov. J. Nucl. Phys. 45 (1987) 292; 47 (1988) 511 ;

E. Eichten and B. Hill, Phys. Lett. B 234 (1990) 511;

H. Georgi, Phys. Lett. B 240 (1990) 447;

B. Grinstein, Nucl. Phys. B 339 (1990) 253;

A. Falk, H. Georgi, B. Grinstein and M. Wise, Nucl. Phys. B 343 (1990) 1.

[12] N. Isgur and M. Wise, Phys. Lett. B 232 (1989) 113; B 237 (1990) 527

[13] J.D. Bjorken, preprint SLAC-PUB-5389 (1990).

[14] H. Georgi, B. Grinstein and M. Wise, Phys. Lett. B 252 (1990) 456;

A. Falk, B. Grinstein and M. Luke, preprint HUTP-90A044 (1990);

M. Luke, Phys. Lett. B 252 (1990) 447.

[15] T. Inami and C.S. Lim, Prog. Theor. Phys. 65 (1981) 297, 1772.

[16] Paricle Data Group, J.J. Hernández et al., Review of particle properties, Phys. Lett. B 239 (1909) 1.

[17] E691 Collab., J.C. Anjos et al., Phys. Rev. Lett. 62 (1989) 1587; preprint FERMI LAB PUB-90/124-E.

[18 ] MARK III Collab., Z. Bai et al., preprint SLAC-PUB-5341, UIUC-HEPG-90-69 (1990).

[19] W. Hou, R. Willey and A. Soni, Phys. Rev. Lett. 58 (1987) 1608. 